\title{
Tuberculin response in preterm infants after BCG vaccination at birth
}

Neonatal Department, Mafraq Hospital, PO Box 2951, Abu Dhabi, United Arab Emirates M R Sedaghatian K Kardouni

Correspondence to: Dr Sedaghatian.

Accepted 24 March 1993

\author{
M R Sedaghatian, K Kardouni
}

\begin{abstract}
A total of 101 preterm infants between 26 and 37 weeks' gestation who received BCG vaccination at birth were evaluated between two and four months after vaccination. Altogether $32 \%$ of these infants had no visible BCG scar. All infants were then tested with tuberculin purified protein derivative (PPD) but only 70 returned for the test to be read 48-72 hours later. The test was negative in 22 $(31 \%)$ and there was an induration of $\leqslant 5$ $\mathrm{mm}$ in another $26(37 \%)$ of the infants. Of 22 infants with no BCG scar, $19(86 \%)$ had an induration of $\leqslant 5 \mathrm{~mm}$. In infants with a positive BCG scar a significantly higher number had an induration of PPD $>5$ $\mathrm{mm}$. There were no significant differences between the rate of scarring and tuberculin conversion in the infants born before or after 32 weeks' gestation. It is considered that routine BCG vaccination at birth on preterm infants is not indicated until a much larger study has been performed.
\end{abstract}

(Arch Dis Child 1993; 69: 309-311)

The efficacy of BCG vaccination and its protection against tuberculosis in the full term infant after birth has been shown by several studies. ${ }^{1-3}$ BCG vaccination is recommended by the World Health Organisation for control of the disease in countries with a high incidence of tuberculosis. ${ }^{4}$ This policy is not routinely applied in preterm infants who may be at greater risk of infection than term infants. To the best of our knowledge there are no data on the effectiveness of BCG vaccination in preterm infants of less than 32 weeks' gestation and similar information on preterm babies of more than 32 weeks is limited. ${ }^{5}$

The purpose of this study was to evaluate the effectiveness of giving BCG vaccination to preterm infants by looking at the following criteria. (1) The percentage of negative scars. (2) The percentage of tuberculin conversion between two and four months later. (3) The number of complications of the vaccination.

\section{Subjects and methods}

During a period of 18 months from November 1989 to December 1992, 289 preterm infants whose gestational ages were confirmed by the Dubowitz scoring system ${ }^{6}$ were given BCG vaccination in the first week of life. The vaccine used was lyophilised BCG vaccine from the Behring Laboratories in the Federal
Republic of Germany. A homogenous suspension in a concentration of $0.5 \mathrm{mg} / \mathrm{ml}$ was made and $0.1 \mathrm{ml}$ given intradermally in the right deltoid region.

Altogether 101 of these preterm infants (26-37 weeks' gestation) returned to the neonatal clinic between two and four months after vaccination. Infants were excluded from the study only if they had severe congenital anomalies. The site of the vaccination was evaluated and graded as follows: no scar, or a papule of 1-2 mm, 3-4 mm, 4-5 $\mathrm{mm}$, or $>5$ $\mathrm{mm}$. The tuberculin purified protein derivative (PPD) test was then performed on the middle third of the flexor surface of the forearm. The PPD was purified lyophilised tuberculin from the Institute Merieux, Lyon, France. The intradermal injection was performed by a single nurse with $0.1 \mathrm{ml}$ of the solution, which contained 10 IU of purified tuberculin. The maximum transverse diameter of induration was measured with a transparent plastic ruler 48-72 hours after the test. The PPD test was graded as follows: non-reactive $(\leqslant 1 \mathrm{~mm}$ induration) or an induration of $2-5 \mathrm{~mm}, 6-9$ $\mathrm{mm}$, or $\geqslant 10 \mathrm{~mm}$.

\section{Results}

Of the 101 preterm infants who returned to the hospital for evaluation of the vaccination site no scar was found in 32, a papule of 1-2 mm in five, $3-4 \mathrm{~mm}$ in $16,4-5 \mathrm{~mm}$ in 31 , and $>5 \mathrm{~mm}$ in 17 (table 1). The range of scar was $1-18 \mathrm{~mm}$ (mean $3.2 \mathrm{~mm}$ ).

All these infants were tuberculin PPD tested but only 70 returned for the test to be read. Of these 70 infants no BCG scar was found in 22 and a scar found in 48 on initial evaluation of the vaccination site. The PPD test was negative in $22(31 \%)$, an induration of $2-5 \mathrm{~mm}$ was found in $26(37 \%), 6-9 \mathrm{~mm}$ in $16(23 \%)$, and $\geqslant 10 \mathrm{~mm}$ in six $(9 \%)$. The range of induration was $0-15 \mathrm{~mm}$ (mean $4.1 \mathrm{~mm}$ ) (see fig 1 ). Of the 22 with no scar and 48 with a scar, 13 (59\%) and nine (19\%) respectively had a nonreactive tuberculin PPD test (fig 2). There was a significantly higher reactive PPD test among those with a visible scar $\left(\chi^{2}=9.5967\right.$, $p=0.0019)$. An induration of $>5 \mathrm{~mm}$ on PPD test was found in $19(39 \%)$ infants with a visible scar in contrast to three $(14 \%)$ with no scar $(p=0.0583$ ) (fig 2). Table 1 compares the BCG scar in infants $\leqslant 32$ or $>32$ weeks' gestation and table 2 the tuberculin PPD induration; there was no statistically significant difference. When the rate of BCG scar and tuberculin conversion were compared between our previous study in full term infants and the present 


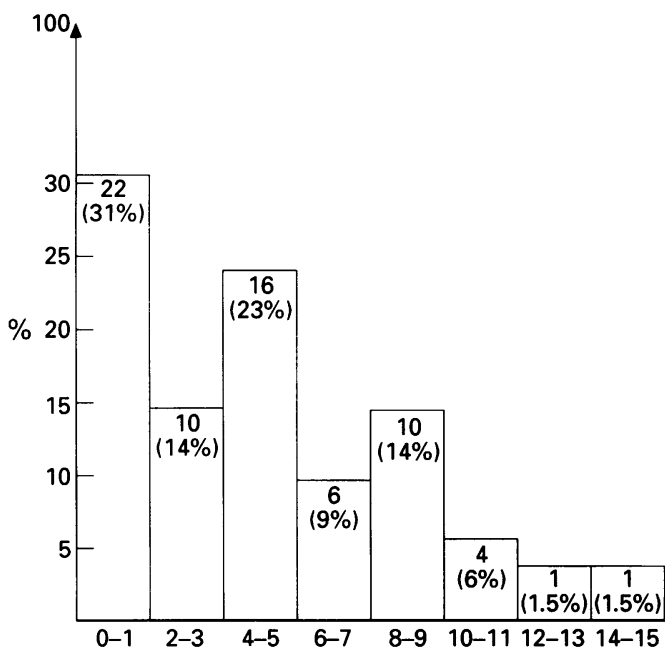

Figure 1 Induration diameter of the PPD test $(\mathrm{mm})$ in 70 preterm infants.

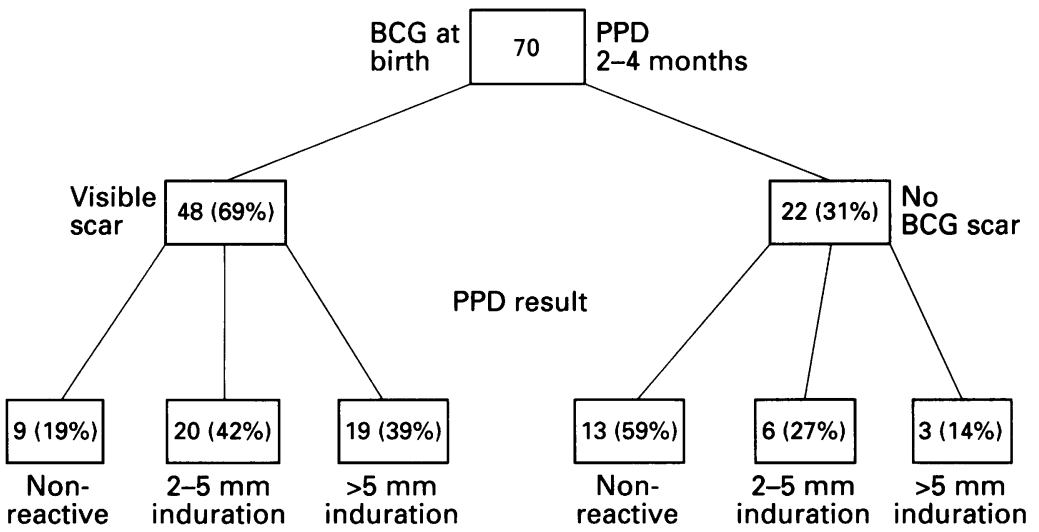

Figure 2 Evaluation of BCG scar in 70 preterm infants.

study in preterm infants (table 3), both were significantly higher in term infants $(p<0.001)$.

\section{Discussion}

In many developing countries tuberculosis is still a major public health problem. The use of vaccine at birth in full term infants has been shown to protect against the haematogenous spread of primary tuberculosis. ${ }^{7} 8$ The efficacy and safety of vaccination in preterm infants has not been well established. To the best of our knowledge there is only one study on 12 preterm infants of more than 32 weeks' gestation that was carried out in Nigeria. ${ }^{5}$ There was $83 \%$ tuberculin conversion with an induration $>5 \mathrm{~mm}$ after BCG vaccination at birth. This study excluded all infants who were small for gestational age and who had sepsis, jaundice, respiratory distress syndrome, apnoea, or congenital malformation. The rate of tuberculin conversion with an induration of $>5 \mathrm{~mm}$ in our study was only $31 \%$. This was $18 \%$ for preterm infants between $26-32$ weeks' gestation and $38 \%$ for those between 33-36 weeks'. We did not exclude any babies because of respiratory distress syndrome, ventilatory support, jaundice, or apnoea; the only exclusions were for major congenital anomalies. The presence of a BCG scar has been used as one of the criteria to assess the coverage of vaccina- tion. The absence of scar has been found to be between $3-25 \%$ in different studies in term infants..$^{10}$ and less than $10 \%$ in preterm infants. ${ }^{5}$ In this study $32 \%$ of the infants had no BCG scar and there was a significant difference for tuberculin conversion between infants with and without a BCG scar. When we compared the numbers of those with a scar and the numbers of those with a reactive PPD test between the present study and our previous study in term infants, ${ }^{1}$ the full term infants had a significantly higher number of BCG scars and tuberculin conversion (table 3). Although the size of the tuberculin reaction after vaccination is not generally thought to influence the degree of protection offered by BCG, the nonreactive tuberculin test should be tested by a higher concentration of PPD (100 IU). We did not observe any complications of the vaccine such as lymphadenopathy or sterile abscess in the preterm infants. This could be due to the low response of preterm infants to BCG vaccination.

Our study indicates that giving BCG vaccination to preterm infants at birth results in a relatively high percentage of infants with no BCG scar $(32 \%)$ and a non-reactive tuberculin PPD test $(31 \%)$. We did not exclude our sick infants from the study, as most preterm infants of less than 32 weeks' gestation need ventilatory assistance and antibiotics; this

Table 1 Comparison of BCG scar in 101 preterm infants at $\leqslant 32$ and $>32$ weeks' gestation; * results are number (\%)

\begin{tabular}{lcc}
\multicolumn{3}{c}{ Gestation (weeks) } \\
& $26-32(n=34)$ & $33-36(n=67)$ \\
& $2 \cdot 6$ & $3 \cdot 4$ \\
Mean scar size (mm) & $0-8$ & $0-18$ \\
$\quad$ Range & & \\
Size of papule (mm) & $11(32)$ & $21(31)$ \\
$\quad$ No scar & $1(3)$ & $4(6)$ \\
1-2 & $8(24)$ & $8(12)$ \\
$3-4$ & $10(29)$ & $21(31)$ \\
$4-5$ & $4(12)$ & $13(20)$ \\
$>5$ & &
\end{tabular}

Difference between groups: $\chi^{2}=3 \cdot 1467, \mathrm{p}=0 \cdot 5336$.

Table 2 Comparison of PPD test induration in 70 preterm infants at $\leqslant 32$ and $>32$ weeks' gestation; * results are number (\%)

\begin{tabular}{lll}
\hline & \multicolumn{2}{c}{ Gestation (weeks) } \\
\cline { 2 - 3 } & $26-32(n=22)$ & $33-36(n=48)$ \\
\hline Mean induration size (mm) & $3 \cdot 0$ & $4 \cdot 6$ \\
$\quad$ Range & $0-10$ & $0-15$ \\
Size of induration $(\mathrm{mm})^{\star}$ & $9(41)$ & $13(27)$ \\
$\quad \leqslant 1 \dagger$ & $9(41)$ & $17(35)$ \\
$\quad 2-5 \dagger$ & $4(18)$ & $18(38)$ \\
$>5$ &
\end{tabular}

†Non-reactive.

Difference between groups: $\chi^{2}=2 \cdot 8314, \mathrm{p}=0 \cdot 2428$.

Table 3 Comparison of BCG scar and PPD induration in term and preterm infants

\begin{tabular}{|c|c|c|c|}
\hline & $\begin{array}{l}\text { Term } \\
\text { infants } \\
\text { (1987) }\end{array}$ & $\begin{array}{l}\text { Preterm } \\
\text { infants } \\
\text { (1992) }\end{array}$ & Significance \\
\hline $\begin{array}{l}\text { Total No infants } \\
\text { No BCG scar }(\%) \\
\text { No returned for }\end{array}$ & $\begin{array}{l}387 \\
30(8)\end{array}$ & $\begin{array}{r}101 \\
32(32)\end{array}$ & $\begin{array}{l}\chi^{2}=39 \cdot 23 \\
\mathrm{p}<0 \cdot 0001\end{array}$ \\
\hline $\begin{array}{l}\text { test to be read } \\
\text { Non-reactive PPD (\%) }\end{array}$ & $\begin{array}{l}264 \\
37 \text { (14) }\end{array}$ & $\begin{array}{l}70 \\
22(31)\end{array}$ & $\begin{array}{l}\chi^{2}=10.3692 \\
p<0.001\end{array}$ \\
\hline
\end{tabular}


may have partly influenced our results. The numbers of infants in our study, as well as in our previous study, are not large enough to draw any firm conclusions. Therefore a control study with a large number of infants should be performed to establish whether routine administration of BCG vaccine in preterm infants at birth is warranted.

The authors are grateful to Dr Gamil Absoud for statistical analysis and $\mathrm{Mr}$ Johnson Pokkath for secretarial assistance.

1 Sedaghatian MR, Shana'a AK. Evaluation of BCG at birth in the United Arab Emirates. Tubercle 1990; 71: 177-80.

2 Ferguson RG, Simes AB. BCG vaccination of Indian infants in Saskatchewan. Tubercle 1949; 30: 5-11.

3 Rosenthal SR, Loewinsohn E, Graham ML, Liveright D, Thom MG, Johnson V. BCG vaccination against tuber- culosis in Chicago. A twenty years study statistically analyzed. Pediatrics 1961; 28: 622-41.

4 World Health Organisation. BCG vaccination policies. WHO Tech Rep Ser 1980: 652.

5 Dawadu AH. Tuberculin conversion following BCG vaccination in pre-term infants. Acta Paediatr Scand 1985; 74: 564-7.

6 Dubowitz LMS, Dubowitz V, Goldberg C. Clinical assessment of gestational age in the newborn infant. $\mathcal{f}$ Pediatr ment of gestation

7 Curtis HM, Leck I, Bomford FN. Incidence of childhood tuberculosis after neonatal BCG vaccination. Lancet 1984; 1: 145-8.

8 Padungchan S, Konjanart S, Kasiratta $S$, et al. The effectiveness of BCG vaccination of the newborn against childhood tuberculosis in Bangkok. Bull World Health Organ 1986; 64: 247-58.

9 Karalliede S, Katugaha LP, Uragoda CG. Tuberculin response of Sri Lanka children after BCG vaccination at birth. Tubercle 1987; 68: 33-8.

10 Grindulus H, Bayham MI, Scott PH, Thompson RA, Wharton BA. Tuberculin response two years after BCG vaccination at birth. Arch Dis Child 1984; 59: 614-9. 